\title{
К ВОПРОСУ О СООТНОШЕНИИ РЕЛЯТИВИСТСКОИ И КЛАССИЧЕСКОЙ МЕХАНИКИ
}

\section{Введение}

Принято думать, что взаимоотношение между релятивистской и классической механикой исчерпывается предельным переходом $c \rightarrow \infty(c-$ скорость света) в духе принципа соответствия. На самом деле один лишь предельный переход недостаточен. Он не ликвидирует релятивистского тождества массы и энергии и неспособен привести к нерелятивистскому понятию энергии (см. $\left.\left[{ }^{1,2}\right]\right)$. Оказывается необходимой добавочная операция - вычитание релятивистской энергии покоя, что приводит к правильному нерелятивистскому понятию энергии. После этого мы получаем, по-видимому, полностью все величины и соотношения нерелятивистской механики.

В определенном ограниченном смысле это действительно так. Но мы хотим обратить в настоящей статье внимание на возможность более широкой трактовки вопроса. В результате мы придем к выводу, что предельный переход $c \rightarrow \infty$, даже в сочетании с вышеупомянутой вычитательной операцией, не выражает адекватно соотношения между релятивистской и классической механикой.

Начнем с общих соображений. Недостаток предельного перехода $c \rightarrow \infty$ состоит прежде всего в том, что он носит формальный характер и, в связи с этим, нефизичен по отношению к свету. В самом деле, конечность скорости света не есть специфически релятивистский факт; скорость света была измерена задолго до создания теории относительности. Полагая скорость света бесконечной, мы, следовательно, игнорируем эти измерения и вводим своеобразное световое дальнодействие. Но для этого нет никаких оснований. Из того, что гравитация в классической теории Ньютона обладает свойством дальнодействия, не вытекает, что тем же свойством должны обладать любые другие взаимодействия: нельзя считать дальнодействие всеобщим принципом классической механики. В частности, скорости тел в классической механике всегда конечны (хотя могут быть сколь угодно большими). Поэтому мы не согласны с распространенным мнением (см., напр., $\left[{ }^{3}\right]$, с. 13), будто основное отличие релятивистской теории от классической состоит в том, что в последней возможна мгновенная сигнализация, тогда как в первой скорость сигнала имеет верхний предел, равный скорости света. Мгновенных сигналов в классической теории не существует, как не существует их и в теории относительности. В них нет и нужды, ибо введение абсолютного 
времени и обоснование всей вообще классической механики возможно при определенных предпосылках без всякой мгновенной сигнализации.

Против приведенных соображений можно, казалось бы, возразить, что световые явления к механике (а тем более к классической) никакого отношения не имеют и что, следовательно, с точки зрения чистой механики нет нужды считаться с фактической конечностью скорости света. Однако это далеко не так. Свет является носителем массы, энергии и импульса, и его взаимодействие с телами должно во многих случаях учитываться наряду с чисто механическими взаимодействиями. В релятивистской механике так и делается, но те же механические свойства света должны сохраняться и в нерелятивистском пределе, поскольку в противном случае из механики выпадает широкая и практически важная область. Нет оснований считать эту область исключительно релятивнстской, так как, повторяем, скорость света конечна не только в релятивистской, но и в нерелятивистской физике и так как импульс, масса и энергия света не являются специфически релятивистскими понятиями. Подчеркнем, что классическая теория не требует представления о свете, как о «чистой энергии», хотя такое понимание было в свое время широко распространено. Свет и в классической физике может быть наделен массой и импульсом; между тем, предельный переход $c \rightarrow \infty$ обращает обе эти величины в нуль.

Другое возможное возражение таково. В классической механике масса тела не зависит от скорости, а в релятивистской механике масса выражается формулой

$$
m=m_{0}\left(1-v^{2} / c^{2}\right)^{-1 / 2}
$$

где $m_{0}$ - масса покоя, а $v$ - скорость тела. Чтобы сделать массу в этой формуле не зависящей от скорости, единственной возможностью является, очевидно, предельный переход $c \rightarrow \infty$. Отсюда, казалось бы, однозначно следует, что перейти от релятивистской механики к классической невозможно иначе, как только приняв скорость света бесконечной.

Однако и это возражение несостоятельно, так как нет нужды исходить из формулы (1). Ниже покажем подробно, что переход от релятивистской механики к классической можно выполнять превентивным в отношении этой формулы способом. Аналогичные соображения относятся и к формулам сокращения длин, замедления времени, сложения скоростей, к преобразованиям Лоренца и ко всем прочим релятивистским соотношениям.

\section{Роль основных постулатов теории относительности}

Для того, чтобы переход от релятивистской механики к классической не был чисто формальным, необходимо провести его на уровне основных постулатов теории относительности. Главную роль играет здесь принцип постоянства скорости света. Отказавшись от него и подчинив скорость света обычному классическому правилу сложения скоростей, мы и приходим к классической механике. Здесь могут сослаться на то, что бесконечная скорость света тоже удовлетворяет тому же классическому правилу. Это верно, и именно в силу этого предельный переход $c \rightarrow \infty$ приводит к классической механике. Однако этот переход требует слишком много, больше того, что строго необходимо для обоснования классической механики. Поэтому классическая механика получается этим способом урезанной - из нее, как сказано выше, выпадает важная область - взаимодействие света с телами. С другой стороны, бесконечная скорость света подчиняется и релятивистскому правилу сложения 
скоростей; поэтому предельный переход $c \rightarrow \infty$ сохраняет второй постулат Эӥнштейна. Сохраняется также принцип относительности. Следовательно, устремляя скорость света к бесконечности, мы идем на своеобразный компромисс: мы получаем классическую механику, но оставляем в силе основные положения теории относительности. Ценой этого компромисса является, опять-таки, отказ от описания механических взаимодействий света с телами. Нам эта плата представляется слишком высокой - тем более, что платить приходится в сущности за призрак, за номинальное сохранение исходных постулатов теории относительности, без сохранения самой теории, на них основанной. Наоборот, естественным и последовательным методом является содержательный отказ от второго постулата Эйнштейна, оставляющий скорость света конечной. В последующих пунктах рассмотрим с этой точки зрения некоторые узловые вопросы.

\section{Взаимодействие света с телами}

Ранее было показано [4,5], что вывод релятивистской зависимости массы от скорости возможен наиболее простым образом путем применения законов сохранения массы и импульса к процессу поглощения телом светового потока. Этот способ, кроме простоты, ценен еще тем, что в нем непосредственно используется второй постулат Эйнштейна. Это обстоятельство позволяет легко и наглядно продемонстрировать, каким образом отказ от второго постулата приводит к классической независимости массы от скорости. Тем самым становится понятным, что для этого вовсе не нужно полагать скорость света бесконечной.

Напомним вывод, о котором идет речь. Пусть на неподвижное тело массы покоя $m_{0}$ падает световой поток со скоростью $c$, массой $\mu$ и импульсом $\mu c$. В результате полного поглощения света тело получает скорость $v$ и масса покоя его тоже изменяется. В силу законов сохранения массы и импульса

$$
\begin{aligned}
\mu+m_{0} & =m_{1} \gamma(v), \\
\mu c & =v m_{1} \gamma(v),
\end{aligned}
$$

где $m_{1}$ - масса покоя тела после поглощения света, а $\gamma(v)$ - множитель, определяющий зависимость массы от скорости. В инерциальной системе, в которой тело покоится после поглощения света, аналогично нмеем:

$$
\begin{aligned}
\mu^{\prime}+m_{0} \gamma(v) & =m_{1}, \\
\mu^{\prime} c^{\prime} & =v m_{0} \gamma(v),
\end{aligned}
$$

где $\mu^{\prime}$ и $c^{\prime}-$ масса и скорость света в этой системе. Исключая из формул (2) $\mu$ и из формул (3) $\mu^{\prime}$, находим:

$$
\begin{aligned}
& m_{0}=m_{1}(1-v / c) \gamma(v), \\
& m_{1}=m_{0}\left(1+v / c^{\prime}\right) \gamma(v) .
\end{aligned}
$$

Перемножая эти формулы, получаем

$$
\gamma(v)=\left[(1-v / c)\left(1+v / c^{\prime}\right)\right]^{-1 / 2},
$$

а подставляя последнее выражение в одну из тех же формул, имеем

$$
m_{1}=m_{0}\left(1+v / c^{\prime}\right)^{1 / 2}(1-v / c)^{-1 / 2} .
$$

Наконец, из вторых формул (2) и (3) с учетом (6) находим

$$
\mu^{\prime}=\mu\left(c / c^{\prime}\right)(1-v / c)^{1 / 2}\left(1+v / c^{\prime}\right)^{-1 / 2} .
$$


Все дальнейшее зависит теперь от $c^{\prime}$. Если положим $c^{\prime}=c$, то получим релятивистские формулы:

$$
\begin{gathered}
\gamma(v)=\left(1-v^{2} / c^{2}\right)^{-1 / 2}, \\
\mu^{\prime}=\mu(1-v / c)^{1 / 2}(1+v / c)^{-1 / 2}, \\
m_{1}=m_{0}(1+v / c)^{1 / 2}(1-v / c)^{-1 / 2} .
\end{gathered}
$$

Но если хотим получить соответствующие нерелятивистские соотношения, то, считая начальный покой тела абсолютным, мы должны принять

$$
c^{\prime}=c-v,
$$

где $c$ - изотропная скорость света в абсолютно покоящейся системе. Тогда формулы (5)-(7) дадут:

и

$$
\begin{gathered}
\gamma(v)=1, \\
\mu^{\prime}=\mu
\end{gathered}
$$

$$
m_{1}=m_{0}(1-v / c)^{-1} \text {. }
$$

Из этих формул только первые две согласуются с формулами (8) и (9) в их предельном виде при $c \rightarrow \infty$. Но формула (14) не получается из соответствующей релятивистской формулы (10) при том же предельном переходе. Отметим, что это отличие непосредственно связано с обращением массы света при $c \rightarrow \infty$ в нуль. В самом деле, если во вторых уравнениях (2) и (3) положим $c \rightarrow \infty$, то получим $\mu=\mu^{\prime}=0$. Но если масса света равна нулю, то, понятно, масса поглотившего свет тела измениться не может, т. е. должно быть $m_{1}=m_{0}$, что и дает формула (10) в ее предельном виде. Если же будем считать скорость света в нерелятивистском случае конечной, то масса света будет отлична от нуля, что и влечет за собой изменение массы тела при поглощении света, согласно формуле (14).

Нам могут по поводу формул (10) и (14) возразить, что они всетаки согласуются друг с другом, если отбросим члены второго и высших порядков относительно $v / c$, но сохраним члены первого порядка. Это верно, но тогда нарушится соответствие между формулами (9) и (13). Отсюда мы должны заключить, что не существует вообще такого автоматического правила типа предельного перехода, которое превращало бы релятивистские формулы в нерелятивистские. Это обстоятельство мы и имели в виду, говоря, что переход следует осуществлять превентивным относительно готовых формул способом. Переход состоит просто в отказе от второго постулата Эйнштейна и в замене его классическим правилом сложения скоростей, причем все формулы должны выводиться заново. Мы показали эффективность этого метода в вопросе о механическом взаимодействии света с телом.

\section{Энергия света}

Другой узловой вопрос касается энергии света. Энергия света W связана с его массой формулой

$$
W=\mu c^{2},
$$

совпадающей по виду с общим релятивистским соотношением между массой и энергией. Однако здесь мы трактуем эту формулу вне связи с законом эквивалентности массы и энергии. Раз мы включаем свет в систему классической механики, то его массу и энергию следует рас- 
сматривать не как тождественные друг другу величины, а просто как взаимосвязанные, подобно тому как в классической формуле

$$
T=m v^{2} / 2
$$

взаимосвязаны масса тела и его кинетическая энергия $T$.

Теперь возникает вопрос: возможно ли на деле включить формулу (15) в систему классической механики? Не должна ли классическая энергия света быть вдвое меньше, т. е.

$$
W=\mu c^{2} / 2
$$

подобно классической кинетической энергии тела? Чтобы ответить на эти вопросы, вспомним, что кинетическая энергия ни в теории относительности, ни в классической теории не является единственным видом энергии. Любое тело обладает также внутренней энергией. Даже элементарной частице можно приписать в классической теории произвольно любую внутреннюю энергию, поскольку классическая энергия вообще определяется с точностью до произвольной аддитивной постоянной. Поэтому мы вправе наделить и свет, в согласии с электромагнитной теорией, энергией $\mu c^{2}$, из которой половина является кинетической энергией света, а другая половина - его внутренней энергией. Следовательно, формула (15) не противоречит возможности нерелятивистской теории с конечной скоростью света.

Вышесказанное подлежит уточнению. Мы должны считать внутреннюю энергию света инвариантной величнной, т. е. равной $\mu c^{2} / 2$ в любой инерциальной системе. Это согласуется с классической инвариантностью внутренней энергии тел, а также с инвариантностью массы притом не только тел, но, согласно формуле (13), и самого света. Кинетическая же энергия света равна $\mu c^{\prime 2} / 2$, где $c^{\prime}$ равно $c$ только, вообще говоря, в абсолютно покоящейся системе.

Чтобы продемонстрировать согласие этих соображений с классической механикой, опишем, например, с энергетической точки зрения процесс поглощения света в теле, рассмотренный в предыдущем пункте. Прежде всего из формул (2) находим:

$$
v=c \mu\left(m_{0}+\mu\right)^{-1}
$$

(отметим, что эта формула является одинаково и релятивистской, и нерелятивистской). Обозначив начальную внутреннюю энергию тела через $U_{0}$ и конечную через $U_{1}$, напишем формулу баланса энергии в ннерциальной системе начального покоя тела:

$$
\mu c^{2}+U_{0}=U_{1}+\left(m_{0} v^{2} / 2\right)(1-v / c)^{-1}
$$

(здесь учтена формула (14)) и в инерциальной системе конечного покоя тела (с учетом формулы (13)):

$$
\mu c^{2} / 2+(\mu / 2)(c-v)^{2}+U_{0}+m_{0} v^{2} / 2=U_{1} .
$$

После подстановки в эти формулы, согласно (18), $c \mu\left(m_{0}+\mu\right)^{-1}$ вместо $v$, обе они дадут одно и то же выражение для $U_{1}$ :

$$
U_{1}=U_{0}+\left(\mu c^{2} / 2\right)\left(2 m_{0}+\mu\right)\left(m_{0}+\mu\right)^{-1} .
$$

Таким образом, введение в нерелятивистскую теорию понятия массы света не исключает одновременного использования понятия энергии света, в согласии с известной уже в дорелятивистской электродинамике формулой (15). Сохраняя скорость света конечной, мы получаем возможность сохранить также оба фундаментальных и универсальных понятия классической механики - понятия массы и энергии в их взаимо- 
связи, не ограничивая нигде их применимости. Тем самым с новой ясностью выявляется роль теории относительности в отношении этих понятий: не взаимосвязь массы и энергии, как часто думают, дает она, но, показывая их полную тождественность (см. $\left.\left[{ }^{6,7}\right]\right)$, объединяет их в едином понятии «массэргии» (см. $\left[{ }^{8}\right]$, с. 189$)$.

\section{Заключительные замечания}

В качестве дополнительного довода в пользу сохранения конечности скорости света в нерелятивистской механике укажем на метод обоснования теории относительности без использования второго постулата Эйнштейна. Вместо него в основу берется требование групповости преобразований. В $\left[{ }^{9}\right]$ этот метод был изложен в таком виде, что независимо от теории относительности получалась наряду с-ней и нерелятивистская теория как альтернативный, логически возможный вариант, не нуждающийся в бесконечности скорости света.

Подчеркнем, далее, что, показывая логическую возможность нерелятивистской механики с конечной скоростью света, мы далеки от мысли считать эту теорию способной заменить теорию относительности. Но в области малых скоростей эта теория дает все-таки результаты, близкие к релятивистским. В этом смысле, подобно «чистой» нерелятивистской механике, она могла бы иметь некоторое реальное значение. Отметим, что, как уже выше было указано, результаты этой теории согласуются с релятивистскими частью в первом порядке относительно $v / c$, частью же лишь в нулевом порядке. Так, например, формула преобразования массы света согласуется в нулевом порядке (см. (9) и (13)), а формулы преобразования импульса $p$ и энергии $W$ света - в первом порядке. В самом деле, из $p=\mu c$ и $p^{\prime}=\mu^{\prime} c^{\prime}$ следует, с учетом формулы (13),

$$
p^{\prime}=p(1-v / c)
$$

(нерелятивистская формула), тогда как согласно теории относительности

$$
p^{\prime}=p(1-v / c)^{1 / 2}(1+v / c)^{-1 / 2} .
$$

Для нерелятивистской энергии света имеем

$$
W^{\prime}=W / 2+(W / 2)(1-v / c)^{2} .
$$

Отсюда

$$
W^{\prime}=W\left(1-v / c+v^{2} / 2 c^{2}\right),
$$

что в первом порядке (и даже, акцидентально, во втором!) согласуется с релятивистской формулой

$$
W^{\prime}=W(1-v / c)^{1 / 2}(1+v / c)^{-1 / 2} .
$$

Дело, однако, не в этой близости теорий или, по крайней мере, не столько в этом. Нет фактически большого смысла применять нерелятивистскую теорию даже в области малых скоростей, поскольку теория относительности работает в этой области в смысле простоты ничуть не хуже, а в смысле точности лучше. Главная ценность выяснения взаимоотношения между релятивистской и классической механикой лежит в области методики. Как было показано в [ $\left.{ }^{5}\right]$, применение понятий массы и импульса света, вводимых независимым от теории относительности образом, чрезвычайно облегчает и упрощает обоснование релятивистской механики. Но это и означает, что, приступая к построению релятивистской механики, мы должны располагать основными понятиями нереляти- 
внстской механики не в узком смысле механики тел, но в более широком понимании, включающем механику света. В наш век лазеров и в будущем веке фотонных ракет такое расширение старого понятия представляется более чем естественным.

Напоследок еще одно замечание. М. Бунге в $\left[{ }^{10}\right]$, касаясь вопроса о взаимоотношениях теорий, справедливо отмечает неоднозначность соответствия между нерелятивистскими и релятивистскими теориями. Частично его аргументация совпадает с нашей. С одним заключением М. Бунге все-таки нельзя согласиться. Он утверждает, что электромагнитная теория Максвелла для пустого (лишенного зарядов) пространства не имеет нерелятивистского предела, так как предельный переход $c \rightarrow \infty$ дает только статическую теорию, а никаких иных скоростей теория вообще не содержит. Это все так, но это означает просто ограниченность физического смысла предельного перехода, о чем мы уже говорили. Нерелятивистский аналог релятивистской теории электромагнитного поля в пустоте все-таки существует. Он получается в результате отказа от постулатов Эйнштейна. Уравнения Максвелла, не содержащие никакой иной скорости, кроме скорости света, сохраняют тогда такой вид только в абсолютно покоящейся инерциальной системе. При переходе в другую инерциальную систему на основе преобразований Галилея уравнения Максвелла несколько изменяют свою форму, причем в них появляется и абсолютная скорость этой системы (см. [ $\left.\left.{ }^{11}\right]\right)$. Скорость света в этой, логически мыслимой, теории остается конечной и подчиняется классическому правилу сложения скоростей.

\section{Л И ТЕРА Т У Р А}

1. Кард П. Г. Принципы относительности и тождественности в теории относительности. В кн.: Физическая наука и философия, М., 1973, с. 266.

2. К а рд П. Г., Уч. зап. ТГУ, вып. 360, 21 (1975)

3. Л анд а у Л. Д., Л и фши и Е. М., Теория поля, Изд. 6-е, М., 1973.

4. K a r d P., Nõukogude Kool, nr. 2, 144 (1974).

5. К а р д П., Изв. АН ЭССР, Физ. Матем., 24, 305 (1975)

6. K a r d P., Erirelatiivsusteooria II, TRU rotaprint, Tartu, 1972, § 16.

7. К а рд П. Г., Уч. зап. ТГУ, вып. 331, 3 (1974).

8. Д жем м е р М., Понятие массы в классической и современной физике, М., 1967

9. К а рд П., Изв. АН ЭССР, Физ. Матем., 24, 449 (1975)

10. Бунге M., Философия физики, М., 1975, с. $259-267$.

11. Ka rd P., Erirelatiivsusteooria I, TRU rotaprint, Tartu, 1972, § 2.

Тартуский государственный университет
Поступила в редакцию

11/VII 1975

\section{P. KARD}

\section{RELATIVISTLIKU JA KLASSIKALISE MEHAANIKA VAHEKORRAST}

Et valguse mass ja impulss ei ole oma olemuselt relativistlikud mōisted, peavad nad säilima ka relativistlikult mehaanikalt klassikalisele ülemineku korral. Järelikult peab valguse kiirus $c$ jääma sel puhul lōplikuks, sest muidu osutuvad valguse mass ja impulss nulliks Eleminek $c \rightarrow \infty$ taastab küll ka klassikalise mehaanika, ent lünklikuna, sest sel juhul ei ole võimalik kirjeldada valguse ja kehade vastastikust mehaanilist mõju. Seetōttu tuleb ülemineku puhul loobuda Einsteini teisest postulaadist ning allutada lõplik valguse kiirus klassikalisele kiiruste liitmise seadusele. Valguse energiat tuleb sel korral vaadelda kahe osa - kineetilise energia ja (invariantse) siseenergia summana. Selline kehade ja valguse klassikaline mehaanika on vastuolutu. Tulemustelt ühtib ta relativistliku mehaanikaga nullinda, esimese või teise järgu täpsusega $v / c$ suhtes ( $v$ on vaadeldava inertsiaalsüsteemi absoluutne kiirus). Nii ühtib valguse massi teisendusvalem relativistliku teisendusvalemiga nullindas järgus, impulsi teisendusvalem esimeses järgus ja energia teisendusvalem teises järgus. Kuigi sel klassikalisel teoorial vaevalt saab olla praktilisi rakendusi (kas vōi ligikaudse teooria mõttes), on tal sịski suur metoodiline väärtus. 


\section{P. KARD}

\section{ZUR FRAGE VON DEN GEGENSEITIGEN BEZIEHUNGEN ZWISCHEN DER RELATIVISTISCHEN UND DER KLASSISCHEN MECHANIK}

Da die Begriffe der Masse und des Impulses des Lichtes ihrem Wesen nach keine relativistischen Begriffe sind, müssen sie beim Ubergang von der relativistischen zur klassischen Mechanik erhalten bleiben. Also muß dabei die Lichtgeschwindigkeit $c$ ihre Endlichkeit bewahren, damit Masse und Impuls des Lichtes nicht gleich Null werden. Durch den Grenzübergang $c \rightarrow \infty$ erhält man zwar auch die klassische Mechanik, doch ist sie unvollständig, da sie die mechanische Wechselwirkung des Lichtes mit den Körpern nicht zu beschreiben vermag. Deswegen muß man beim Ubergang zur klassischen Mechanik das zweite Postulat Einsteins durch das klassische Additionstheorem der Geschwindigkeiten (angewandt auf die endliche Lichtgeschwindigkeit) ersetzen. Die Energie des Lichtes läßt sich dann als Summe des kinetischen und inneren (invarianten) Anteils darstellen. Eine solche klassische Mechanik der Körper und des Lichtes ist widerspruchsfrei. Ihre Ergebnisse stehen mit denen der relativistischen Mechanik größenordnungsgemäß in Einklang. So ist die Transformationsformel für die Masse des Lichtes (bezüglich der Größe $v / c$, wo $v$ die absolute Geschwindigkeit des Inertialsystems ist) auf die nullte, die für den Impuls auf die erste und die für die Energie auf die zweite Ordnung genau. Obgleich diese klassische Theorie kaum praktischen Anforderungen (wenn auch angenähert) gerecht werden kann, hat sie doch bedeutsamen methodischen Wert. 